\title{
Über den Resorptionsweg der Purinkörper.
}

\author{
Von \\ Joh. Biberfeld und Jul. Schmid. \\ (Aus dem pharmakologischen Institute und der medizinischen Poliklinik der Universität \\ Breslau.) \\ (Der Redaktion zugegangen am 28. April 1909.)
}

Unter den im Darm zur Resorption gelangenden Stoffen spielen die Nucleinkörper, über deren kompliziertes weiteres Schicksal im Tierkörper wir in den letzten Jahren exaktere Kenntnisse gewonnen haben, eine bedeutsame Rolle. Wir wissen, daß die mit der Nahrung aufgenommenen Nucleoproteide im Magen bereits eine Spaltung in Nuclein und Eiweiß erfahren, und daß im Darm unter der Einwirkung des Pankreassekrets eine weitere Spaltung des Nucleins in Nucleinsäure und Eiweiß erfolgt. Hier macht aber der fermentative Spaltungsprozeß zunächst Halt ${ }^{1}$. Würde schon im Darmtraktus eine weitere Spaltung stattfinden, so wäre die Resorption der Nucleinsubstanz stark eingeschränkt, denn die aus dem weiteren Abbau der Nucleinsäure zunächst sich ergebenden Purinbasen sind in freiem $\mathrm{Zu}$ stand der Resorption nur mangelhaft zugängig (Krüger und $\left.\mathrm{Schmid}^{2}\right)$ ). Eine Veränderung geht, wie Abderhalden und Schittenhelm gefunden haben, die Nucleinsäure im Darm nur in der Hinsicht noch ein, als sie leicht löslich und dialysabel wird. Was nun mit der in die Darmwand aufgenommenen Nuclein-

1) Im Pankreasextrakt ist zwar von verschiedenen Autoren (Araki, Nakajama und besonders Fr. Sachs, diese Zeitschr., Bd. XLVI, S. 337) eine Nuclease ebenso wie in anderen Organen (Schittenhelm, ebenda Bd. XLII) nachgewiesen worden, doch hat Sachs gezeigt, daß diese Nuclease durch Trypsin und Alkalien zerstört wird; er spricht ihr daher nur intracelluläre Bedeutung zu.

2) Diese Zeitschrift, Bd. XXXIV, S. 549. 
säure zunächst weiter geschieht, wissen wir nicht. Es ist zwar nach den Untersuchungen letzterer Autoren ${ }^{1}$ ) bekannt, daß die Darmschleimhaut ein die Nucleinsäure zersetzendes Ferment, sowie auch die zur Bildung von $\bar{U}$ führenden Fermente besitzt, aber wir können nicht behaupten, daß die resorbierte Nucleinsäure vor ihrer Weiterbeförderung und ihrer Verwendung als Aufbaumaterial in der Darmschleimhaut diesen fermentativen Umsetzungen total oder auch nur teilweise anheimfällt. Weiter ist uns auch der Transportweg für die Nucleinsäure oder deren Spaltprodukte vom Darm aus in den Körper durchaus unbekannt. Wir können von vornherein vermuten, daß diesen Weg die Blutgefäße bilden, weil dies für die meisten Stoffe (Eiweiß, Kohlenhydrate, Wasser, Salze) zutrifft. Allerdings müssen wir daran erinnern, daß bei Zufuhr abnorm großer Mengen von diesen Stoffen ein kleiner Teil auch seinen Weg durch den Ductus thoracicus wählt. Umgekehrt wissen wir ja auch vom Fett, daß dieses bei übermäßig großer Aufnahme in die Darmschleimhaut teilweise von den Blutkapillaren, also nicht ausschließlich von den Chylusgefäßen aufgenommen wird.

Zunächst war die Frage zu entscheiden: auf welchem Weg wird die Nucleinsäure resp. deren Spaltprodukte forttransportiert, nachdem sie die Darmwand passiert haben?

Dieser Frage sind wir durch Untersuchungen der Lymphe von Katzen und Hunden nach verschiedenartiger Fütterung nähergetreten. - Zur Methodik bemerken wir folgendes: Die aus dem Ductus thoracicus gewonnene Lymphe wurde zwei Stunden (meist noch etwas mehr) mit dem gleichen Volumen 3\%iger Schwefelsäure am Rückflußkühler gekocht, alkalisch gemacht und noch einmal aufgekocht, mit Essigsäure enteiweißt und das Filtrat mit Bisulfit-Kupfersulfat, meist auch noch mit Silberlösung auf Purine untersucht. - Im Harn wurde die Harnsäure nach Krüger-Schmid, der Gesamt-N nach Kjeldahl bestimmt.

In der Literatur fanden wir keine Angaben über einen etwaigen Puringehalt normaler Lymphe. Wir schickten des-

1) Diese Zeitschrift, Bd. XLVII, S. 452. Die Versuche wurden mit dem Extrakt des Rinderdarms angestellt. 
halb einige Versuche an unbehandelten Tieren voraus, von denen folgende angeführt seien.

Versuch I. Katze, $2400 \mathrm{~g}$, erhält $2 \mathrm{Uhr} 500 \mathrm{ccm}$ Milch. Um 4 Uhr Operation in Paraldehydnarkose. $4^{1 / 2}-6 \mathrm{Uhr}\left(2^{1 / 2}\right.$ bis 4 Stunden der Verdauungsperiode) werden 12,6 ccm Lymphe aufgefangen; Purine sind darin nicht nachzuweisen.

Versuch III. Katze, $3200 \mathrm{~g}$, erhält am 13. XI. ausschließlich Pferdefleisch zu fressen (500 g); am 14., $7 \mathrm{Uhr}$, noch $250 \mathrm{~g}$ Fleisch; 113/4 Uhr Operation in Paraldehydnarkose. In $1^{1 / 2}$ Stunden werden $9,8 \mathrm{ccm}$ Lymphe aufgefangen; Purine sind darin nicht vorhanden.

Normale Lymphe enthält sonach weder bei purinfreier noch bei purinreicher Kost Purine.

Aber auch nach Verfütterung von Nucleinsäure, ${ }^{1}$ ) also nach Überschwemmung mit purinlieferndem Materiale, traten in der Katzenlymphe keine Purine auf, z. B.:

Versuch V. Katze, $3300 \mathrm{~g}$, erhält 1 Uhr 3,0 g Nucleinsäure (in etwas Soda gelöst) mit Milch per os, säuft auch nachher noch Milch (im ganzen ca. $500 \mathrm{ccm}$ ). Von $4^{1 / 2}$ bis 5 Uhr werden $8,2 \mathrm{ccm}$ Lymphe aufgefangen, die sich als purinfrei erweisen.

Das gleiche Resultat erhielten wir auch am Hunde, wie folgende Versuche zeigen:

Versuch VI. Hund, $8000 \mathrm{~g}, 11 \mathrm{Uhr} 10,0 \mathrm{~g}$ Nucleinsäure mit $250 \mathrm{~g}$ gehacktem Pferdefleisch per os. Von 4-7 Uhr in Morphinnarkose 22,1 ccm Lymphe aufgefangen (etwas blutig gefärbt). Purine nicht vorhanden.

Versuch VII. Hund, $12000 \mathrm{~g}, 10 \mathrm{Uhr}$ 15,0 g Nucleinsäure in $500 \mathrm{ccm}$ Milch, $1 \mathrm{Uhr}$ nochmals $500 \mathrm{ccm}$ Milch. Von 4-6 Uhr $27 \mathrm{ccm}$ Lymphe (sehr fettreich und schnell gerinnend) aufgefangen; enthält keine Purine.

Versuch IX. Hund, $9000 \mathrm{~g}$. Erhält $7^{3 / 4} \mathrm{Uhr}$ einen Liter Milch. 11 Uhr 0,09 Morph. hydroch. subcutan.

1) Die Nucleinsäure haben wir für die meisten Versuche aus Kalbsthymus selbst dargestellt; in einigen Versuchen benutzten wir das Mercksche animale Präparat. 
12 Uhr 10 Min. bis 12 Uhr 25 Min. ca. 12 ccm Lymphe.

> 1 \ 15 . $30 \mathrm{ccm}$ Lymphe (noch sehr fettreich).

1 Uhr 15 Min. 10,0 g Nucleinsäure in $500 \mathrm{ccm}$ Milch (mit etwas Soda gelöst) mit der Schlundsonde.

1 Uhr 15 Min. bis 2 Uhr 25 Min. 22,5 ccm Lymphe (fettreich)

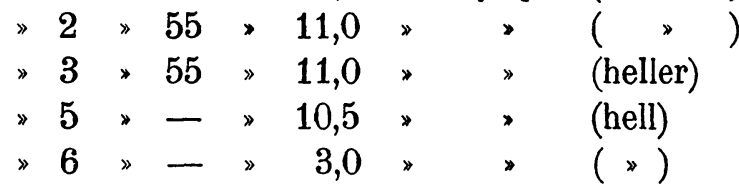

In keiner der Lymphportionen waren Purine nachweisbar.

Wir haben in den angeführten Versuchen die in der Zeit von $3^{1} / 2-7^{1 / 2}$ Stunden nach Einbringung der Nucleinsäure sezernierte Lymphe purinfrei gefunden. Nun wären gegen dieses Resultat die Einwände möglich, daß die von uns gefütterte Nucleinsäure überhaupt nicht oder erst viel später, als wir die Lymphe prüften, resorbiert werde. Trotzdem diese Einwände an sich schon sehr wenig wahrscheinlich sind, haben wir sie noch durch die folgenden Versuche widerlegt.

Versuch X. Hund, 7700 g. 10. III., 9 Uhr, Anlegung einer Hypospadie proximal vom Penisknochen (nach G. Rosenfeld), um besser katheterisieren zu können; das Tier frißt den Tag über nichts.

Bis 11. III., 91/2 Uhr, $180 \mathrm{ccm}$ Urin mit $\mathrm{N}=4,648 \mathrm{~g}$ und $\overline{\mathrm{U}}=0,0275 \mathrm{~g}$.

Bis 12. III., 9 $1 / 2$ Uhr (ebenfalls Hunger), $108 \mathrm{ccm}$ Urin mit $3,338 \mathrm{~g} \mathrm{~N}$ und $0,032 \mathrm{~g} \overline{\mathrm{U}}$.

Am 12., 91/2 Uhr, frißt der Hund 500 g gehacktes Pferdefleisch; bis 13. III., 91/2 Uhr, $470 \mathrm{ccm}$ Urin mit 10,234 g N und $0,010 \mathrm{~g} \overline{\mathrm{U}}(?)$.

Am 13. III., 91/2 Uhr, erhält er $500 \mathrm{~g}$ Pferdefleisch $+10 \mathrm{~g}$ Nucleinsäure; bis $12^{1 / 2} \mathrm{Uhr}, 23 \mathrm{ccm}$ Urin mit $0,441 \mathrm{~g} \mathrm{~N}$ und $0,01 \mathrm{~g} \overline{\mathrm{U}} ;$ bis $3^{1 / 1_{2}} \mathrm{Uhr} 102 \mathrm{ccm}$ Urin mit $1,792 \mathrm{~g} \mathrm{~N}$ und $0,063 \mathrm{~g} \overline{\mathrm{U}}$; bis $6 \mathrm{Uhr} 134 \mathrm{ccm}$ Urin mit $2,413 \mathrm{~g} \mathrm{~N}$ und $0,070 \mathrm{~g} \overline{\mathrm{U}}$; bis 14 . III., $9^{1 / 2} \mathrm{Uhr}, 380 \mathrm{ccm}$ Urin mit $5,016 \mathrm{~g} \mathrm{~N}$ 
und $0,136 \mathrm{~g} \overline{\mathrm{U}}$; erhält $500 \mathrm{~g}$ Fleisch; bis 15. III., 91/2 Uhr, $595 \mathrm{ccm}$ Urin mit $12,684 \mathrm{~g} \mathrm{~N}$ und $0,158 \mathrm{~g} \overline{\mathrm{U}}$.

In diesem Versuche ist also bereits in der Urinportion, die von der 4.-7. Stunde nach der Fütterung ausgeschieden wurde, eine erhebliche Vermehrung der Harnsäure zu konstatieren. Daß diese nur auf die gefütterte Nucleinsäure bezogen werden kann, ist ohne weiteres klar.

Wir haben auch an ein und demselben Tiere die Ausscheidungsverhältnisse der Nucleinsäure im Urin und in der Lymphe verfolgt.

Versuch XI. Hund, 8000 g, mit Hypospadie. Vom 29. III., $10 \mathrm{Uhr}$ an, kein Futter; Wasser ad libitum. 31. III., $10 \mathrm{Uhr}$, Blase entleert; vom 31.-1. IV., $10 \mathrm{Uhr}, 168 \mathrm{ccm}$ Urin mit $1,981 \mathrm{~g} \mathrm{~N}$ und $0,002 \mathrm{~g} \overline{\mathrm{U}}$.

Am 1. IV. $375 \mathrm{~g}$ gehacktes Pferdefleisch.

Bis 2. IV., $10 \mathrm{Uhr}, 430 \mathrm{ccm}$ Urin mit $12,317 \mathrm{~g} \mathrm{~N}$ und $0,042 \mathrm{~g} \overline{\mathrm{U}} ;$ am 2 . IV. $375 \mathrm{~g}$ gehacktes Pferdefleisch.

Bis 3. IV., $9 \mathrm{Uhr}, 665 \mathrm{ccm}$ Urin mit 12,202 $\mathrm{g} \mathrm{N}$ und $0,057 \mathrm{~g} \overline{\mathrm{U}}$; am 3. IV., $9 \mathrm{Uhr}, 375 \mathrm{~g}$ gehacktes Pferdefleisch + $10 \mathrm{~g}$ Nucleinsäure.

Bis 12 Uhr $90 \mathrm{ccm}$ Urin mit $2,101 \mathrm{~g} \mathrm{~N}$ und $0,064 \mathrm{~g} \overline{\mathrm{U}}$

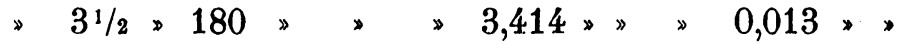

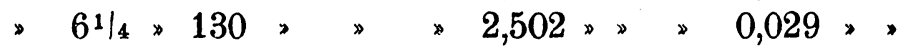

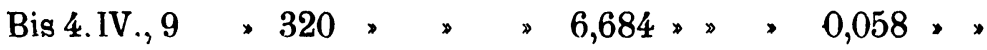
Am 4. IV., 9 Uhr, 375 g Fleisch.

Bis 4. IV., $3 \mathrm{Uhr}, 440 \mathrm{ccm}$ Urin mit $4,497 \mathrm{~g} \mathrm{~N}$ und $0,043 \mathrm{~g} \overline{\mathrm{U}}$

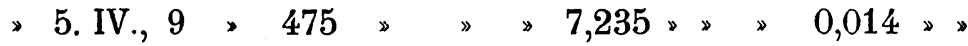

Am 5. VI., 9 Uhr, $375 \mathrm{~g}$ Fleisch.

Bis 6. VI. $9 \mathrm{Uhr}, 1170 \mathrm{ccm}$ Urin mit $12,4 \mathrm{~g} \mathrm{~N}$ und $0,043 \mathrm{~g} \overline{\mathrm{U}}$. Am 6. VI., $9 \mathrm{Uhr}, 375 \mathrm{~g}$ Fleisch $+10 \mathrm{~g}$ Nucleinsäure; 1 Uhr 30 Min. Morphin subcutan.

2 Uhr 55 Min. bis 3 Uhr 25 Min. 21 ccm Lymphe

\begin{tabular}{|c|c|c|c|c|c|}
\hline 4 & » & 25 & » & 46 & ^ \\
\hline 4 & 》 & 55 & » & 21,5 & ע \\
\hline 5 & 》 & 40 & » & 40 & ఎ \\
\hline 7 & * & - & $\nu$ & 26 & » \\
\hline
\end{tabular}


In keiner der Lymphportionen sind Purine nachweisbar.

Dieser Versuch beweist, daß die Resorption der Nucleinsäure und die Ausscheidung ihrer Abbauprodukte sehr bald beginnen kann und erst in 24 Stunden im wesentlichen beendet ist. Das Auffangen der Lymphe in unseren Versuchen fällt demnach in die Zeit, in welcher eine starke Resorption der Nucleinsäure vor sich geht.

Wir sind nach dem Ausfall unserer Untersuchung zu dem Schlusse berechtigt, daß die Purine nicht auf dem Lymphwege resorbiert werden.

Die Ergebnisse unserer Versuche sind:

1. Die Lymphe von Hunden und Katzen enthält weder bei Milch- noch bei Fleischnahrung Purine.

2. Auch bei reichlicher Zufuhr von animaler Nucleinsäure sind keine Purine in der Lymphe vorhanden.

3. Die Ausscheidung der Abbauprodukte verfütterter animaler Nucleinsäure beginnt frühzeitig und ist innerhalb von 24 Stunden im wesentlichen beendet, wie das Verhalten von Harnsäure und Gesamtstickstoff anzeigt. 